\title{
The role of glucose-6-phosphate dehydrogenase in the evolution of longevity in Drosophila melanogaster
}

\author{
L. S. Luckinbill,* \\ V. Riha, \\ S. Rhine* and \\ T. A. Grudzien $\dagger$
}

\author{
* Department of Biological Sciences, \\ Wayne State University, Detroit MI, 48202, U.S.A. \\ $\uparrow$ Department of Biological Sciences, \\ Oakland University, Rochester MI, 48309, U.S.A.
}

Different polymorphic elements of the enzyme glucose-6-phosphate dehydrogenase (G6PD) are favoured under selection for long versus short life span. Replicate independently selected populations of short-lived individuals exhibit a more rapidly migrating and less actively staining allozyme, while long-lived populations have a slower migrating and more active allozyme. These correspond to the common $Z w^{A}$ and $Z w^{B}$ variants of the G6PD locus $Z w$. In vitro measurements show G6PD activity varies with allozymes and life span. Long-lived males have 64 per cent greater activity in G6PD, while females of long-lived strains are 108 per cent higher than those of short-lived strains. Previous studies of these strains have repeatedly demonstrated additivity of life span in $F_{1}$ crosses. Activity of G6PD in reciprocal $F_{1}$ populations is additive and intermediate between parents.

\section{INTRODUCTION}

By the addition of explicit evolutionary content to the earlier demographic concepts advanced by A. Lotka (1925), Fisher (1930) and Haldane (1941) initiated theoretical consideration of the evolution of the components of life history. Since then, theoretical works by Medawar (1952), Cole (1954), Williams (1957), Hamilton (1966) and Charlesworth (1980) have proceeded rapidly to address a variety of related issues, both general and specialized, that include among them the evolution of life span itself.

The validation and testing of theory consisted for a time primarily of empirical comparisons of evidence from life historical studies of natural populations. But more recently, independent studies with a priori testing have demonstrated the validity of an important premise central to the theory of life history evolution; that age-specific success in reproduction determines the increase or decrease in life span. In testing this idea, first Wattiaux (1968), followed by Rose and Charlesworth (1981) and then Luckinbill et al. (1984), Rose (1984) and Luckinbill and Clare (1985), showed that under selection for reproductive success at early or late ages in life, life span respectively either remained short or increased in Drosophila populations. Selection applying opposite patterns of age-specific reproductive success produced, in independent instances, replicate populations with substantial genetically based differences in their life span.

These experiments demonstrate both the validity and direct applicability of evolutionary theory to our understanding of life history in general but they imply very little, within their directed scope, about the identity of the genes or the physiologi$\mathrm{cal} /$ biochemical process(es) by which life span is extended. They do, however, clearly allow the opportunity for such studies. Toward that end, subsequent experiments with selected strains have examined various features other than lifespan affected by selection as a result of either pleiotropy or linkage, and the implications of those findings for the function and location of putative adaptive loci.

Such experiments have included findings by Rose and Charlesworth (1981) and later Rose (1984), Luckinbill et al. (1984) and Luckinbill et al. (1987) demonstrating pleiotropic action of genes for longevity on fecundity. The increased longevity of populations selected for late life reproduction is associated with a decline of fecundity in early life, as Williams' (1957) theory of pleiotropic gene action predicts.

One especially unique feature of these selectively bred stocks has been shown in studies by 
Clare and Luckinbill (1985) and Luckinbill and Clare (1985), who found that crosses of long- with short-lived parental populations are additive and intermediate in life span between parents. Experiments of those studies also demonstrate the existence of a powerful gene-environment interaction, deriving from the developmental environment. When long-lived or $F_{1}$ populations are raised at a very low population density of ten larvae per vial or less (numbers of developing larvae are usually between one and two orders of magnitude higher than this), the longevity of eclosing adults becomes reduced. That is, the phenotype of adult flies is short-lived regardless of their genetic capability. This gene-environment effect results in substantial reductions in life span and may have artifactually confounded several previous experimental attempts at selecting for increased life span in Drosophila that failed (Lints and Hoste, 1974; Lints et al., 1978; Flannagan, 1980). It has been independently verified by Service et al. (1988).

Another set of significant traits were found in studies by Service et al. (1985) and Service (1987) who showed long-lived strains selected in their laboratory to be highly resistent to starvation, desiccation and ethanol fumes. Earlier Doan (1960) followed by Clark and Doan (1983), in studying the adipose mutant of $D$. melanogaster, found that the increased synthesis and storage of lipid in that mutant confers resistance to both starvation and desiccation. However, it was not until Service (1987) that long-lived strains were demonstrated to have both a greater rate of synthesis and total store of lipid. Based on those findings, the physiological mechanism by which life span is improved, was postulated to depend on the metabolism and/or synthesis of lipid.

Luckinbill and Graves (1988) and Graves et al. (1988) have, however, discovered yet a different feature of the physiology associated with divergence in life span of selected stocks. Individuals of long-lived populations have been found to fly, while tethered, an average of 300 per cent longer than those of short-lived populations and as Graves et al. (1988) have shown, the increased duration of flight does not occur as a result of a compensatory reduction in the rate of flight. The duration of flight in Drosophila has long been known from physiological studies to depend on glycogen stores and bears little direct relation to lipid synthesis (Wigglesworth, 1949). Our findings of increased flight ability, therefore, and the recent verification of the same for strains of $D$. melanogaster developed by M. Rose (Graves and Rose, 1989) show clearly that another physiologi- cal pathway apart from lipid synthesis must also differ in long-lived populations.

Fortunately, not oniy is the physiology of flight reasonably well understood, but also the biochemical genetics. Based on our preceding findings concerning flight duration and drawing on the works of Laurie-Ahlberg et al. (1985), Luckinbill et al. (1989) surveyed isozyme variation and found differences existed between long- and short-lived populations in four enzymes of flight. Phosphoglucomutase (PGM), NAD malate dehydrogenase $(\mathrm{MDH})$ and NADP malic enzyme all showed quantitative differences in enzymatic activity only. But, glucose-6-phosphate dehydrogenase (G6PD), on the other hand, was found to differ qualitatively in long- and short-lived stocks, in both larvae and adults. That study was primarily an exploratory survey, however, and made no further investigation of the variation in either allozymes or enzymatic activity itself.

This study investigates the possibility of G6PD as putatively adaptive. In these experiments we examine the type, extent of variation in replicate stocks and activity of allozymes of G6PD with regard to a possible contributing role for that enzyme in adaptation. And finally, we determine the degree to which enzyme activity in $F_{1}$ crosses corresponds to the additivity found in life span by previous studies.

\section{METHODS}

The selection applied to replicate long- and shortlived strains of this study consisted of simply collecting progeny produced at either early or late ages in adult life (Luckinbill et al., 1984). Both mating and egg laying were continuous in all populations throughout life, but only progeny produced in the first four days of adult life or by the last surviving approximately ten pair of individuals were retained for the next generation. Numbers of developing larvae were high in all treatments of selection and density was uncontrolled. Replicate populations consisted of 50 pairs of individuals per half pint bottle.

The methods of horizontal starch gel electrophoresis, as described in Richmond (1972) and Ayala et al. (1972) and cellulose acetate, as in Hebert and Beaton (1986), were used here to examine variation in allozymes of G6PD. Triscitrate buffer $(p \mathrm{H} \mathrm{7 \cdot 0)}$ and tris-Glycine buffer $(p \mathrm{H} \mathrm{8.5)}$ were used respectively. In our previous survey (Luckinbill et al., 1989), variants in enzymes of flight were detected by horizontal starch gel 
electrophoresis such that long- and short-lived individuals were found to differ in allozymes of G6PD, but neither the extent of quantitative variation among selected lines, nor that specific in particular electrophoretic parameters was investigated there. Since the quantitative study of allozyme variation requires the observation of virtually all existing variants, sequential electrophoresis (Ramshaw et al., 1979) was used here to test for undetected allozymes by varying two parameters especially likely to mask their expression, citrate ion concentration and $p \mathrm{H}$. For this, allozyme variation was examined under conditions in which either $p \mathrm{H}(p \mathrm{H}=7 \cdot 0$ or $8 \cdot 5 \pm 0.5$ respectively) or citrate ion concentration (buffer made at $33.6 \mathrm{~g} /$ litre \pm 10 per cent) was varied. For samples examined using cellulose acetate, $p \mathrm{H}$ and ionic concentration were varied in a similar fashion.

Enzymatic activities were measured as in Clark and Keith (1988). Initial measurements show that activity of G6PD remains relatively constant for at least the first six weeks of life. For assays of activity, therefore, ten groups of ten male or female flies, from one to three weeks of age were homogenized in potassium phosphate buffer and centrifuged at $8000 \times \mathrm{g}$. for $30 \mathrm{~min}$ before measurement of activity at $340 \mathrm{~nm}$. Lowry's (1951) method was used for measurement of total protein at $750 \mathrm{~nm}$ and a new standard curve prepared for every set of activities assayed.

Only flies of the same age and sex were compared. Groups of ten female individuals from replicate three-week-old populations were assayed for enzymatic activity and compared by $t$-test (paired design). Crosses of long- and short-lived populations were reciprocal and each cross consisted of 50 pairs of adults. All other adult parental populations also consisted of 50 pairs of individuals. Activity in $F_{1}$ crosses and their parental (control) populations was assayed at about one week of age.

\section{RESULTS}

In spite of varying the parameters of electrophoresis here, only two allozymes of G6PD were found under all conditions. Fig. 1 shows a composite zymogram comparing those allozymes in replicate independently selected long- and shortlived strains. Of all the individuals examined in short-lived strains $\left(N_{1}=161, N_{2}=90\right) 100$ per cent exhibited an electrophoretically faster migrating, lighter staining allozyme. Corresponding to this,
SHORT - LIVED STRAINS LONG - LIVED STRAINS
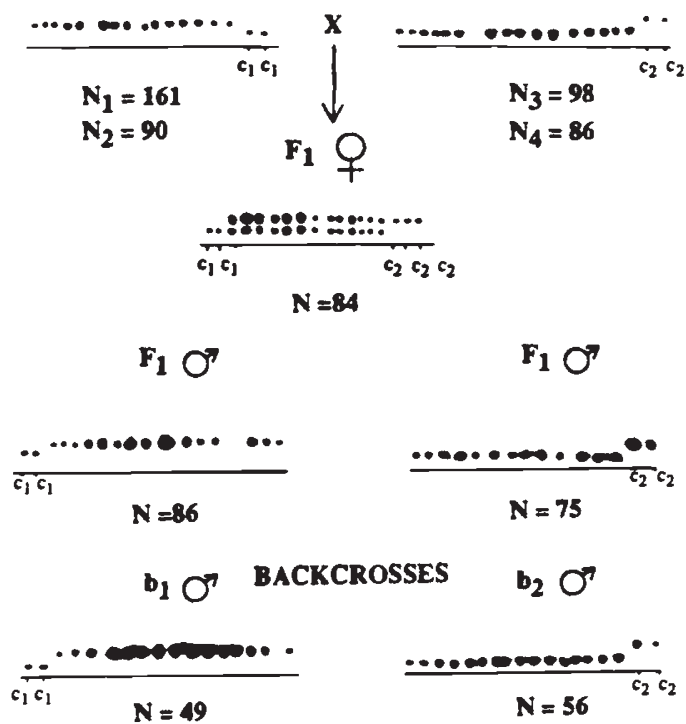

Figure 1 Allozymes of G6PD are shown for replicate longand short-lived populations of $D$. melanogaster, reciprocal $F_{1}$ crosses and backcrosses. Long-lived individuals have the slow migrating $Z w^{s}$ variant, as seen in control $C_{1}$, for comparison with the faster migrating variant $Z w^{f}$ of shortlived populations, as seen in control $C_{2}$. The numbers of individuals assayed in each replicate strain are shown as $N_{1}-N_{4}$. Heterozygous $F_{1}$ females show both $Z w^{\mathrm{f}}$ and $Z w^{s}$ allozymes, while $F_{1}$ males show either band indicating its maternal origin. Backcrosses of $F_{1}$ males to parental populations (directly above each) confirm the location of $Z w^{\mathrm{f}}$ and $Z w^{s}$ as $X$-linked for the same locus.

100 per cent of all long-lived individuals had a slower migrating, intensely active allozyme of G6PD $\left(N_{3}=98, N_{4}=86\right)$. Females of reciprocal $F_{1}$ crosses show both allozymes, while male $F_{1}$ 's show either one, identifying its maternal X-linked origin, with backcrosses concurring.

Eanes (1983) has mapped the structural locus for that gene, called $Z w$ (Zwishenferment) to a position at 62.9 on chromosome 1 . of $D$. melanogaster. The allozymes found in short- and long-lived strains here correspond to the two most common variants, $Z w^{\mathrm{A}}$ and $Z w^{\mathrm{B}}$ for that locus. Williamson and Bentley (1983) have shown that, at low concentrations of NADP, $Z w^{\mathrm{A}}$ and $Z w^{\mathrm{B}}$ code respectively for dimeric and tetrameric forms of G6PD, with a higher molecular weight and lower $K_{\mathrm{m}}$ for G6P in the latter. Our fast migrating variant, designated here as $Z w^{f}$ corresponds to the former $\left(Z w^{\mathrm{A}}\right)$ in its faster rate of migration and reduced activity, while our other variant $Z w^{\text {s }}$, corresponds 
to $Z w^{\mathrm{B}}$. Selection for long- and short-life by the application of opposite age-specific patterns of reproductive success have, therefore, favoured the same respective alleles of G6PD in two independent instances.

Our previous survey (Luckinbill et al., 1989) described the association of a second band with the $Z w^{\text {s }}$ in long-lived individuals. While relatively small numbers of individuals were sampled there, the more extensive examination of this study has shown the occurrance of that very faintly staining, extra fast migrating band to be erratic and follow no predictable pattern of association with $Z w^{\text {s }}$, either in samples of long-lived parents, $F_{1}$ populations, or in segregating backcrosses. But it does appear more often and more intensely in the presence of higher concentrations of mercaptanoethanol and probably results, therefore, from an artifact of preparation.

Even though selective treatments were highly uniform here the resulting longevities vary somewhat between replicates as does the mean duration for tethered flights. The values that populations exhibit under selection are well known to vary among replicates as a function of mutation, the occurrence of particular recombinations, minor environmental differences and random variations in the process of selection itself. Estimates of enzyme activity in particular, vary with such factors as temperature, the extent to which tissue samples are homogenized and the force of centrifugation applied. Comparison of long- and short-lived populations are valid and most critical here, therefore, when applied relatively, contrasting oppositely selected strains of the same replicate set.

Table 1 shows the activity of G6PD at $340 \mathrm{~nm}$ compared within the same sex for individuals from each pair of corresponding strains. Analysis by $t$-test (paired) reveals that long-lived individuals show substantially greater activity in all instances in both males $\left(t_{1}=3.4, t_{2}=3 \cdot 1, P<0.01\right)$ and females $\left(t_{3}=9 \cdot 1, t_{4}=5.0, \quad P<0.001\right)$, for the hypothesis (one-tailed) that greater activity is instrumental in extending life span.

Early studies of the enzyme G6PD associated in vitro differences in activity with differences in allozymes such as we find here. But Cavener and Clegg (1981) found no difference in activity of allozymes when a potassium phosphate homogenization buffer was used instead of tris$\mathrm{HCl}$ and suggested that the use of that buffer may have selectively reduced the activity of one allozyme and artifactually created and observed differences. Miyashita et al. (1986) and Miyashita and Laurie-Ahlberg (1984, 1986), however, found a substantial difference in the activity of these allozyme variants even with the use of potassium phosphate buffer. Our findings here concur strongly with theirs.

In fig. 2 the mean and 95 per cent confidence intervals of activities in samples of reciprocal $F_{1}$ crosses and parental populations (controls) are shown in relation to the midparent value. Ten samples of ten individuals of each population respectively averaged 26.57 (nM NADPH/min/ $\mathrm{mg}$ ) of tissue $(\mathrm{S} . \mathrm{D} .=11.7)$ in short-lived and 54.34 ( $\mathrm{nM} \mathrm{NADPH} / \mathrm{min} / \mathrm{mg}$ ) in long-lived females $($ S.D. $=10 \cdot 7) . F_{1}$ populations averaged 38.91 $(\mathrm{nM} \mathrm{NADPH} / \mathrm{min} / \mathrm{mg})($ S.D. $=13.9)$ for the cross of short-lived males by long-lived females, while females of the reciprocal cross averaged 39.6 $(\mathrm{nM} \mathrm{NADPH} / \mathrm{min} / \mathrm{mg})(\mathrm{S} . \mathrm{D} .=11 \cdot 7)$.

Neither $F_{1}$ population is significantly different from the midparent value $\left(t_{1}=0.35, t_{2}=0.23\right)$. They do, however, differ substantially from both the short- and long-lived parental control populations as the grouping according to StudentNeuman-Kuels test indicates (fig. 2 lower). Reciprocal $F_{1}$ populations are indistinguishable from one another, but differ significantly from the

Table 1 Means and standard deviations for measurements of G6PD activity (nmol NADPH/min/mg protein) in replicate long-and short-lived populations. Comparison by paired $t$-test is shown for activities in ten samples of ten individuals each for a strain. Values shown are averages for all ten samples of each sex.

\begin{tabular}{|c|c|c|c|c|c|c|c|}
\hline & \multicolumn{7}{|c|}{ Enzyme activity $(\mathrm{nmol} / \mathrm{min} / \mathrm{mg})$} \\
\hline & \multicolumn{2}{|c|}{ Long-lived } & \multicolumn{2}{|c|}{ Short-lived } & \multirow{2}{*}{$\begin{array}{l}N \\
\text { (groups of } 10)\end{array}$} & \multirow{2}{*}{$\begin{array}{l}t \\
\text { (paired) }\end{array}$} & \\
\hline & Mean & s.d. & Mean & s.d. & & & \\
\hline Females & $44 \cdot 7$ & $9 \cdot 3$ & $21 \cdot 4$ & $5 \cdot 2$ & 10 & $9 \cdot 14$ & $P<0.001$ \\
\hline Males & $60 \cdot 1$ & $11 \cdot 2$ & $38 \cdot 3$ & $10 \cdot 4$ & 10 & $3 \cdot 39$ & $P<0.010$ \\
\hline Females & $47 \cdot 2$ & $15 \cdot 0$ & $26 \cdot 8$ & 8.7 & 10 & $5 \cdot 00$ & $P<0.001$ \\
\hline Males & $41 \cdot 8$ & $20 \cdot 1$ & $23 \cdot 8$ & $9 \cdot 8$ & 10 & 3.07 & $P<0.010$ \\
\hline
\end{tabular}


MIDPARENT

VALUE

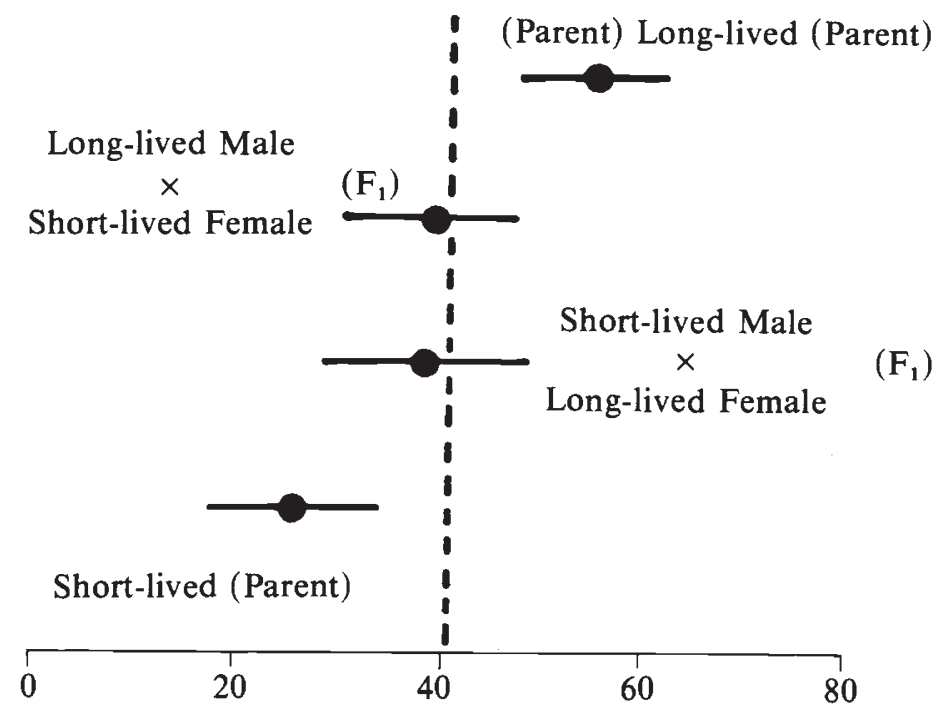

ACTIVITY

(nM NADPH/min./mg tissue)

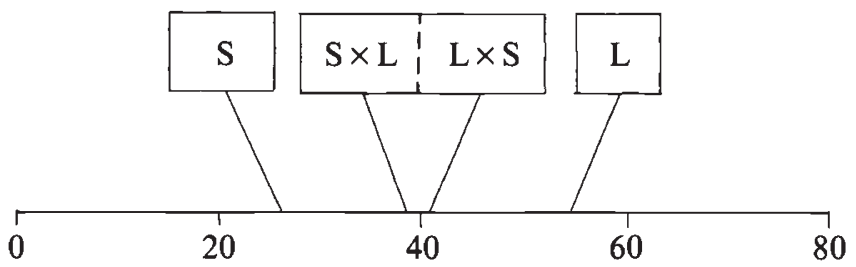

ACTIVITY

(nM NADPH/min./mg tissue)

Figure 2 Compares means and 95 per cent confidence intervals for activity (nmol NADPH/min/mg protein) in $F_{1}$ females of reciprocal crosses with parental strains as controls.

parents. $F_{1}$ populations are additive in activity of the enzyme G6PD, therefore, just as they are in life span.

\section{DISCUSSION}

These experiments show that:

(1) Replicate populations, independently selected for short and long life span exhibit an identical distribution of polymorphic elements of the G6PD locus. All the individuals of both shortlived populations are found to have a fastmigrating, less enzymatically active allozyme designated $Z w^{\mathrm{f}}$, while long-lived populations have a slower migrating more active allozyme $Z w^{s}$. These correspond to two common respective variants at that locus, $Z w^{\mathrm{A}}$ and $Z w^{\mathrm{B}}$. Selection has clearly distinguished, in replicate independent instances, between allozyme variants of the G6PD locus. 
(2) Measures of in vitro activity of G6PD vary with allozymes and life span of strains. All samples from long-lived populations are significantly greater in activity of G6PD. Longlived males exceed those in short-lived populations by 64 per cent, while females are 108 per cent greater.

(3) G6PD activity or reciprocal $F_{1}$ crosses is additive and intermediate between values of parental lines, as has also been shown for longevity. Based on the results of our previous survey (Luckinbill et al., 1989), this study examines the quantitative difference in allozyme frequency and activity at a single locus in replicate oppositely selected populations of $D$. melanogaster. While our prior study indicated that contributing genetic differences may exist in at least three other enzymes, G6PD is the only enzyme for which a qualitative difference in allozymes is evident at this time. Although an undetermined number of other genetic elements may also contribute to the observed difference in longevity in these stocks, the clarity of experimental indications here, propriety of the physiological mechanisms implicated and the correspondence with independent studies by others, suggests this enzyme plays an important role in adaptation.

This study, ascribes the differences observed in the distribution and activity of allozymes to opposite treatments of selection for early and latelife reproduction. In that allozymes of the same locus are considered here, the observed distribution of correlated characters cannot be explained as effects of linkage, but one problem that does require consideration in an experiment of this design is the potential affect of drift on the assortment of alleles observed. Since in each of only two independent instances, the same type of allele has been found to be fixed under the same selective treatment, the problem is in determining whether the observed assortment of allozymes could result from the effects of genetic drift or inbreeding as a consequnce of small population size. Fortunately, existing formulations allow us to make an estimate of the maximum likelihood of that occurrence for an analysis of this question.

To begin with, we must assume an initial frequency of our two alleles $A_{1}$ and $A_{2}$ that is optimal for producing the result observed (that is, two subpopulations fixed after 40 generations at a frequency of $A_{1}=1 \cdot 0$, with two others fixed at a frequency of $A_{2}=1 \cdot 0$ ). The optimal frequency for alleles of the ancestral population to produce this result by the chance effects of drift would, therefore, be $A_{1}=A_{2}=0.5$.
Next, using these values, the proportion of populations homozygous as a result of genetic drift over 40 generations $\left(H_{40}\right)$ can be calculated from the formula of Hartl and Clark (1989):

$$
H_{40}=1-H_{0}(1-1 / 2 N)^{t}
$$

where $H_{0}$ is defined here as the proportion of heterozygotes in the initial population and the value of $H_{0}$ is assumed to equal $0 \cdot 5 .(N)$ is defined as population size. $2 \mathrm{~N}$ would equal 200 here except for the fact that G6PD is sex linked, therefore, $2 N=150 .(t)$ is the number of generations over which drift occurs and here $t=40$. Assuming hypothetical values for $H_{0}$ (and frequencies of genes) most likely to produce our observed result under the null hypothesis of drift, the expected proportion of homozygous subpopulations after 40 generations of drift is $H_{40}=0 \cdot 62$. That is, about 31.0 per cent of such subpopulations might be expected to be fixed for one allele (allozyme) or the other under these circumstances with 41 per cent of subpopulations continuing segregation for both alleles. The probability of drawing four such fixed subpopulations at random is therefore $(0.64)^{4}=0 \cdot 17$ which we call $P_{1}$.

Next, we can calculate the number of possible ways in which four populations can assort under the chance effects of drift for two alleles present in the ancestral population as shown in table 2 (left). Five different ways exist in which four populations can assort with respect to two alleles under drift. But in only one instance are two populations fixed for each allele. Therefore, the probability of observing such an event by chance is one in five. We call this value $P_{2}$, where $P_{2}=0 \cdot 2$. And third, given this distribution of fixed populations we can also calculate the probability of the occurrence by chance of two populations fixed for the same (more active) allozyme under the same treatment of selection as shown in table 2 (right). That is, three configurations exist in which four populations (fixed for two allozymes) are distributed equally between two experimental treatments. But in only one such arrangement are both populations fixed for the more active allozyme under the same treatment of selection, as actually observed. This likelihood is called $P_{3}$ and, therefore, $P_{3}=0 \cdot 33$. And finally, the overall probability that four populations, homozygous as a result of drift in 40 generations, would be fixed by chance for two allozymes as actually observed in experiments we designate $\left(P_{\mathrm{t}}\right)$. The value $\left(P_{\mathrm{t}}\right)$ is calculated from the product of the three independent probabilities $\left(P_{1}\right)\left(P_{2}\right)$ $\left(P_{3}\right)$, where $P_{\mathrm{t}}=(0.17)(0.20)(0.33)=0.011 . P<$ 0.05 , therefore for the likelihood of observing these 
Table 2 At left shows the possible configurations in the distribution of two alleles, $A_{1}$ and $A_{2}$, fixed in four populations by drift. Populations are assumed to descend from a common ancestral population with frequencies of $A_{1}=A_{2}=0 \cdot 5$. In one possibility of five are two populations fixed for each allele as observed in experiments. Table 2 (right) shows possible configurations with two such populations allotted to each treatment of selection, as in the design of this experiment. Where alleles $A_{1}$ and $A_{2}$ differ in activity, only one combination of three corresponds to observed results

Distribution of alleles $A_{1}$ and $A_{2}$ fixed in four populations by drift

\begin{tabular}{lll}
\hline & \multicolumn{2}{l}{ Number of alleles } \\
\cline { 2 - 3 } & $A_{1}$ & $A_{2}$ \\
\hline (1) & 4 & 0 \\
$(2)$ & 0 & 4 \\
$(3)$ & 3 & 1 \\
$(4)$ & 1 & 3 \\
$(5)$ & 2 & 2
\end{tabular}

Distribution of populations fixed for two alleles with different activities in two experimental treatments

Experimental treatments (selection)

\begin{tabular}{ll}
\hline $\begin{array}{l}\text { Early- } \\
\text { reproduction }\end{array}$ & $\begin{array}{l}\text { Late- } \\
\text { reproduction }\end{array}$ \\
$A_{1} A_{1}$ & $A_{2} A_{2}$ \\
$A_{2} A_{2}$ & $A_{1} A_{1}$ \\
$A_{1} A_{2}$ & $A_{1} A_{2}$
\end{tabular}

results by chance. Departures from the assumed initial gene frequencies of 0.5 would reduce the likelihood of observing this distribution still further.

In view of these arguments, the principal determinant of the observed distribution of allozymes among strains and treatments must be regarded as the selection applied for early and late-life reproduction. Under this selection, the latter treatment appears to favour increased longevity in association with the more active polymorphic elements of the G6PD locus. In vitro differences in activity in replicate selected strains correspond to observed differences in allozymes and longevity, as does activity in $F_{1}$ crosses. In view of this, some explanation is required of the adaptive advantage provided by G6PD, physiological features associated with it, and the known genetic basis for production of that enzyme with respect to life span in selected strains.

The first such feature concerns the position of the G6PD locus here and its relationship to longevity in $\mathrm{F}_{1}$ crosses. The locus of G6PD allozymes described here has been mapped to within $0.3 \mathrm{cM}$ to the left of $Z w$, the known structural locus for G6PD, at 62.9 on Chromosome 1 (Rhine, unpublished). But populations of $F_{1}$ crosses are additive and intermediate between oppositely selected parents. How can a sex-linked locus give rise to additivity, such as shown in figs 1 and 3? One answer to this may be found in the fact that production of the enzyme G6PD appears to be under the control of a gene complex comprised of an autosomal element(s) operating in addition to the known structural element. An example of the relative roles of X-linked and autosomal elements is shown by the dosage compensation that occurs in males. G6PD is one of a few genes, in fact, for which dosage compensation is known to occur and has been shown by Ganguly et al. (1985) to result from enhanced transcription. Dosage compensation appears to result in general, from the action of an autosomal modifier(s) on the sex-linked structural gene (Lucchesi, 1973, 1983). Additivity for such an enzyme would be contingent, therefore, on a relatively larger effect of autosomal modifiers, as opposed to the structural element. Selection appears to have acted in this instance to favour variations promoting greater activity by both the more influential autosomal modifier(s) and the $\mathrm{X}$-linked structural locus as well.

Independently of this, Miyashita et al. (1986), in an elegant series of experiments, investigated the genetic basis of activity in the enzymes G6PD and 6PGD, using extracted $\mathrm{X}$-chromosomes and autosomes. Both fast and slow migrating electrophoretic variants of G6PD contributed to significant variation from chromosome 1 , but the effect of autosomes was far more substantial. Segregating allozyme variants of chromosome 1 accounted for about 10 per cent of the total variance, while autosomal background contributed 49-53 per cent. In other experiments, Miyashita and Laurie-Ahlberg (1986) showed that the autosomal effect in the same extracted lines derived primarily from the third chromosome.

The study by Luckinbill et al. (1988) assaying chromosomal effects on life span corresponds qualitatively to Miyashita and Laurie-Ahlberg's (1986) examination of enzymatic activity in G6PD and even compares favourably in the proportional effects of different chromosomes. While Miyashita et al. (1986) attribute respectively 10 per cent and 49-53 per cent variation in G6PD activity to the 


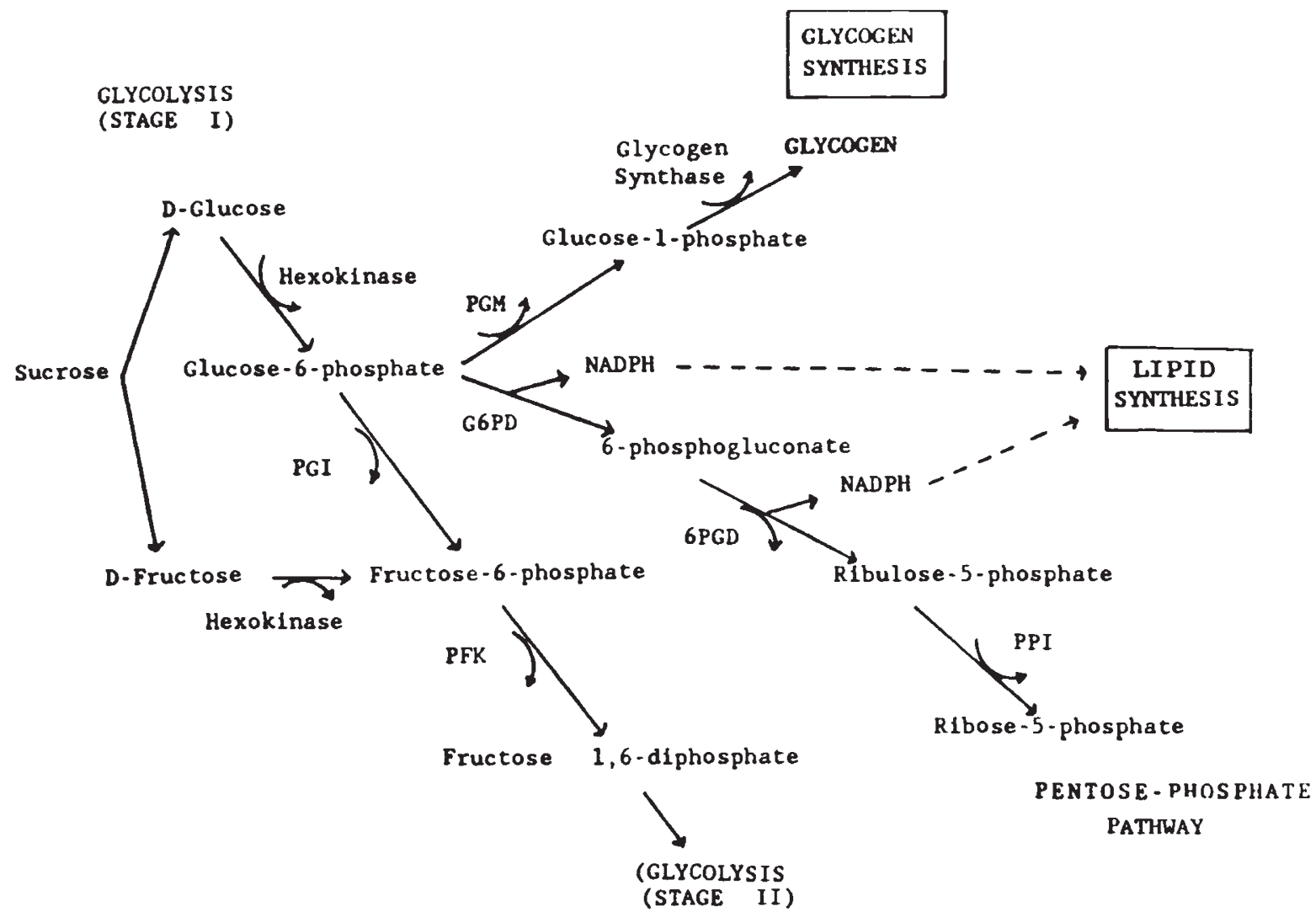

Figure 3 Hydrolyzed sucrose enters the glycolytic pathway as the monosaccharides D-glucose and D-fructose. Increased activity of G6PD in adapted populations is associated with both increased glycogen synthesis and activity in the pentose-phosphate pathway. Increased levels of NADPH from the latter add reducing capacility stimulating synthesis of lipids.

$\mathrm{X}$ chromosome versus autosomal effects, Luckinbill et al. (1988) found that chromosome 1 contributes approximately $28 \cdot 3$ per cent of variation in life span in males, with $45 \cdot 1$ per cent coming from autosomal effects. Effects by chromosome 3 were predominant in both studies. While the correspondance between the identity and proportional effects of linkage groups in these two studies cannot be considered a powerful indication of the association between G6PD and longevity, the additivity of crosses does appear to be explainable here by the preponderant effects of an autosomal gene(s) on life span and G6PD activity, independent of the relationship between those characters.

As a putatively adaptive enzyme, G6PD offers a particularly succinct explanation for the simultaneous occurrence of seemingly unrelated physiological traits correlated with life span. Geer et al. $(1979 a, b)$ and Geer and Laurie-Ahlberg (1984) have shown that adaptation to dietary sucrose in
D. melanogaster increases activity in the enzyme G6PD. As shown in fig. 3, the flow of carbon from sucrose can be diverted from glycolysis by G6PD activity in long-lived individuals, with resulting stimulation of both glycogen synthesis and the pentose-phosphate pathway. One of the principal consequences of pentose-phosphate activity is the production of NADPH, which provides reducing potential to many synthetic processes in $D$. melanogaster, but particularly stimulates synthesis of fatty acids and lipids. Increased glycogen synthesis and pentose-phosphate activity, with greater lipid synthesis deriving from the latter, all occur in association with greater G6PD activity and as such, explain the simultaneous improvement in the associated physiological functions of flight duration and the lipid associated traits; starvation, desiccation and ethanol resistance. All these features, together with increased late-life fecundity may, therefore, derive simultaneously from simple 
adaptation toward more efficient utilization of the sugar sucrose, provided in media. For populations selected for early-reproduction, on the other hand, greater fecundity at an early age is of more importance than long life or persistence to a great age. Under this form of selection, glycogen synthesis and pentose-phosphate activity would assume far less importance and with activity in G6PD reduced, carbon from dietary sucrose would be directed predominantly to glycolysis and egg production.

Although G6PD is often found to be the limiting enzyme for the oxidative branch of the pentosephosphate pathway (Wood, 1985), Cavener and Clegg's (1981) study suggests how the relative importance of variants in G6PD may be determined here in regulating pentose-phosphate activity. Their study found that allozyme variants at the structural locus of G6PD had a slight affect on flux through the pentose-phosphate pathway. This may have resulted from a lack of variation in autosomal modifier(s). However, variants of a functionally related enzyme, 6PGD, had substantial effects, on flux through the pentose-phosphate pathway. Fast and slow migrating variants $6 \mathrm{PGD}^{\mathrm{f}}$ and $6 \mathrm{PGD}^{\mathrm{s}}$, were found for that locus, as well as for G6PD, with the latter, more active form, permitting greater flux in their study. Both replicate long- and short-lived strains of our experiments are fixed for the same slow migrating, active form of $6 \mathrm{PGD}^{\mathrm{B}}$ (not shown), indicating that variants in G6PD may assume importance in regulating pentose-phosphate activity here by default because of insufficient variability in the other regulatory enzyme.

G6PD fits several criteria that earmark a possibly significant role in adaptation. First, is that selection has cleanly differentiated between polymorphic elements of that locus in a way that is inconsistent with sampling error, drift or linkage as alternatives. Next, is that G6PD offers a single unified explanation for several physiologically diverse characters that correlate with life span in adapted strains. And finally is that activity in G6PD behaves as life span does in the genic configurations of $F_{1}$ populations.

While existing evidence presented here supports the possibility that G6PD is adaptive, further testing is essential for a definite understanding of that proposition here. First of all and perhaps most. difficult, is determining the identity and relative contribution of other genetic and biochemical elements, particularly NADP malic enzyme (ME), as it is also responsible for contributing a substantial proportion of the NADPH. It is also important to determine the identity and locality of third chromosome modifier(s) directing transcription of the structural locus of G6PD. And finally, flux in relevant pathways must itself be measured for a thorough evaluation of this adaptive mechanism.

\section{REFERENCES}

AyAla, F. J., POWEll, J. R., TRACEY, M. L., MOURAo, C. A. AND PEREZ-SALAZ, S. 1972. Enzyme variability in the Drosophila willistoni group. IV. Genetic variation in natural populations of Drosophila willistoni. Genetics, 70, 113-139.

CAVENER, D. R. AND CLEGG, M. T. 1981. Evidence for biochemical and physiological differences between enzyme genotypes of Drosophila melanogaster. Proc. Natl Acad. Sci. USA, 78, 4444-4447.

CHARLESWORTH, B. 1980. Evolution In Age-structured Populations. Cambridge University Press.

CLARE, M. J. AND LUCKINBILL, L. S. 1985. The effects of gene-environment interaction on the expression of longevity. Heredity, 55, 19-25.

CLARK, A. G. AND DOAN, W. W. 1983. Desiccation tolerance of the adipose mutant of Drosophila melanogaster. Hereditas, 99, 165-175.

CLARK, A. G. AND KEITH, L. 1988. Variation among extracted lines of Drosophila melanogaster in triacylglycerol and carbohydrate storage. Genetics, 119, 595-607.

COLE, L. C. 1954. The population consequences of life history phenomena. Quart. Rev. Biol., 29, 103-137.

DOAN, W. W. 1960. Developmental physiology of the Mutant Female Sterile (2) Adipose of Drosophila melanogaster. II. Effects of altered environment and residual genome on its expression. J. Exp. Zool., 145, 23-41.

EANES, W. F. 1983. Genetic localization and sequential electrophoresis of Glucose-6-Phosphate Dehydrogenase in Drosophila melanogaster. Biochem. Genetics, 21, 703-711.

FISHER, R. A. 1930. The Genetical Theory of Natural Selection. Clarendon Press, Oxford.

FLANAGAN, J. R. 1980. Detecting early-life components in the determination of the age of death. Mech. Aging Dev., 13, 41-62.

GANGUlY, R., GANGULY, N. AND MANNING, J. E. 1985. Isolation and characterization of the glucose-6-phospate dehydrogenase gene of Drosophila melanogaster. Gene, 35 , 91-101.

GEER, B. W. AND LAURIE-AHLBERG, C. C. 1984. Genetic variation in dietary sucrose modulation of enzyme activities in Drosophila melanogaster. Genet. Res. Camb., 43, $307-$ 321.

GEER, B. W., KROCHKO, D. AND WILlIAMSON, J. H. 1979a. Ontogeny, cell distribution and the physiological role of NADP-Malic Enzyme in Drosophila melanogaster. Biochem. Genetics, 17, 867-879.

GEER, B. W., LINDEL, D. L. AND LINDELL, D. M. $1979 b$, Relationship of the oxidative pentose shunt pathway to lipid synthesis in Drosophila melanogaster. Biochem. Genetics, 17, 881-895.

GRAVES, J. L., LUCKINBILL, L. S. AND NICHOLS, A. 1988. Flight duration and the measurement of wing beat frequency in long- and short-lived Drosophila melanogaster. J. Insect Physiol., 34, 1021-1026. 
GRAVES, J. L. AND ROSE, M. R. 1989. The flight/longevity correlation in Drosophila melanogaster selected for postpońed senescence. In Harrison, D. E. (ed.) Genetic Effects On Aging.

HAldane, J. B. S. 1941. New Paths in Genetics. Allen and Unwin, London.

HAMILTON, W. D. 1966. The moulding of senescence by natural selection. $J$. Theoret. Biol., 12, 12-45.

HARTL, D. AND ClARK, A. G. 1989. Principles of Population Genetics. Sinauer Associates, pp. 78.

HEBERT, P. D. N. AND BEATON, M. 1986. Cellulose Acetate Gel Electrophoresis. University of Windsor, Ontario, Canada, 34pp.

LAURIE-AHLBERG, C. C., BARNES, P. T., CURTSINGER, J. W., EMIGH, T. H., KARLIN, B., MORRIS, R., NORMAN, R. A AND WILTON, A. N. 1985. Genetic variability of flight metabolism in Drosophila melanogaster. II. Relationship between power output and enzyme activity levels. Genetics, $111,845-868$.

LINTS, F. A. AND HOSTE, C. 1974. The Lansing Effect revisited. I. Life-span. Exp. Geront., 9, 51-69.

LINTS, F. A., STOLL, J., GRUWEZ, G. AND LINTS, C. v. 1979. An attempt to select for longevity in Drosophila melanogaster. Gerontology, 25, 192-204.

LOTKA, A. 1925. Elements of Physical Biology. Reprinted in 1956 by Dover Publications N.Y.

LOWRY, O., ROSEBROUGH, N., FARR, A. AND RANDELL, R. 1951. Protein measurements with the Folin phenol reagent. J. Biol. Chem., 193, 265-275.

LUCCHESI, J. C. 1973. Dosage compensation in Drosophila. Ann. Rev. Genetics, 7, 225-237.

LUCHESSI, J. C. 1983. Dosage compensation in Drosophila. Isozymes: Current Topics In Biological and Medical Research Vol. 9: Gene Expression and Development, pp. 179-188.

LUCKINGBILL, L. S., ARKING, R., CLARE, M. J., CIROCCO, W. AND BUCK, S. 1984. Selection for delayed senescence in Drosophila melanogaster. Evolution, 38, 996-1003.

LUCKINBILL, L. S. AND CLARE, M. J. 1985. Selection for life span in Drosophila melanogaster. 1985. Heredity, 55, 9-18.

LUCKINBILL, L. S. AND CLARE, M. J. 1986. A density threshold for the expression of longevity in Drosophila melanogaster. Heredity, 56, 329-335.

LUCKINBILl, L. S., ClARE, M. J., KRELl, W. K., CIROCCO, W. C. AND RICHARDS, P. A. 1987. Estimating the numbers of genetic elements that defer senescence in Drosophila. Evolutionary Ecology, 1, 37-46.

LUCKINBILL, L. S., GRAVES, J. L., REED, A. H. AND KOET. SAWANG, S. 1988a. Localizing genes that defer senescence in Drosophila melanogaster. Heredity, 60, 367-374.

LUCKINGBILL, L. S., GRAVES, J. L., TOMKIU, A. AND SOWIRKA, O. $1988 \mathrm{~b}$. A qualitative analysis of some life history correlates of longevity in Drosophila melanogaster. Evolutionary Ecology, 2, 85-94.
LUCKINBILL, L. S., GRUDZIEN, T. A., RHINE, S. AND WEISMAN, G. 1989. The genetic basis of adaptation to selection for longevity in Drosophila melanogaster. Evolutionary Ecology, 3, 31-39.

MIYASHITA, N. AND LAURIE-AHLBERG, C. 1986. Developmental variation in effects of the second and third chromosomes on the activities of Glucose-6-phosphate and 6Phosphogluconmate dehydrogenases in Drosophila melanogaster. Biochem. Genetics, 24, 447-467.

MIYASHITA, N., LAURIE-AHLBERG, C., WILTON, A. N. AND EMIGH, T. H. 1986. Quantitative analysis of X chromosome effects on the activities of the Glucose-6-phosphate and 6-phosphogluconate dehydrogenases of Drosophila melanogaster. Genetics, 113, 321-335.

MEDAWAR, P. B. 1952. An Unsolved Probelm in Biology. H. K. Lewis, London.

RAMSHAW, A. J., COYNE, J. A. AND LEWONTIN, R. C. 1979. The sensitivity of gel electrophoresis as a detector of genetic variation. Genetics, 93, 1019-1037.

RHINE, S. (unpublished). Quantitative and Mendelian Factors Associated with the Increased Life Span of Selectively Bred Drosophila melanogaster. M.S. Thesis, Wayne State University.

RICHMOND, R. C. 1972. Enzyme variability in the Drosophila willistoni group III. Amounts of variability in the superspecies, D. paulistorum. Genetics, 70, 87-112.

ROSE, M. R. 1984. Laboratory evolution of postponed senescence in Drosophila melanogaster. Evolution, 38, 1004-1010.

ROSE, M. R. AND CHARLESWORTH, B. 1981. Genetics of life history in Drosophila melanogaster II. Exploratory selection experiments. Genetics, 97, 187-196.

SERVICE, P. M. 1987. Physiological mechanisms of increased stress resistance in Drosophila melanogaster selected for postponed senescence. Physiol. Zool., 60, 321-326.

SERVICE, P. M., HUTCHINSON, E. W., MACKINLEY, M. D. AND ROSE, M. R. 1985. Resistance to environment stress in Drosophila melanogaster selected for postponed senescence. Physiol. Zool., 58, 380-389.

SERVICE, P. M., HUTCHINSON, E. W. AND ROSE, M. R. 1988 Multiple genetic mechanisms for the evolution of senescence in Drosophila melanogaster. Evolution, 42, 708-716.

WATtIAUX, J. M. 1968. Cumulative parental age effects in Drosophila subobscura. Evolution, 22, 406-421.

WIGGLESWORTH, v. B. 1949. The utilization of reserve substances in Drosophila during flight. J. Exp. Biol., 26, 150-163.

WILliAMS, G. C. 1957. Pleiotropy, natural selection and the evolution of senescence. Evolution, 11, 398-411.

WILliamSON, J. W. AND BENTLEY, M. M. 1983. Comparative properties of three forms of Glucose-6-phosphate dehydrogenase in Drosophila melanogaster. Biochem. Gen. $21,1153-1166$.

wOod, T. 1985. The Pentose Phosphate Pathway. Academic Press. 